The Astrophysical Journal, 235:749-754, 1980 February 1

(c) 1980. The American Astronomical Society. All rights reserved. Printed in U.S.A.

\title{
OPTICAL OBSERVATIONS OF RADIO JETS
}

\author{
HARVEY R. ButCheR \\ Kitt Peak National Observatory ${ }^{1}$ \\ AND \\ Wil van Breugel and George K. Miley \\ Sterrewacht Leiden, The Netherlands \\ Received 1979 July 17; accepted 1979 August 14
}

\begin{abstract}
Optical observations of the radio jet galaxies 3C $66 \mathrm{~B}, 3 \mathrm{C} 31$, NGC 315, and 3C 449 are reported. Optical counterparts to the inner regions of the radio jets are seen in $3 \mathrm{C} 66 \mathrm{~B}$ and $3 \mathrm{C} 31$, and upper limits are set for optical jets in the other two.

The ratios of optical to radio emission in the jets of $3 \mathrm{C} 66 \mathrm{~B}$ and $3 \mathrm{C} 31$ are only slightly less than that for M87, and the limits set for NGC 315 and 3C 449 are consistent with fairly similar ratios of optical to radio emission. These measurements suggest that a continuous nonthermal spectrum extending from $10^{9}$ to $10^{15} \mathrm{~Hz}$ is a general property of jets. The jet parameters are considered in relation to the intrinsic properties of the galaxies and the associated extended radio emission. Implications for models of energy transport in radio sources are discussed. Significant amounts of interstellar matter are observed in each of the four galaxies, leading us to suggest that models for jets which involve an interaction with the interstellar medium may be most appropriate.
\end{abstract}

Subject headings: galaxies: nuclei — galaxies: structure - radio sources: galaxies

\section{INTRODUCTION}

Over the past several years a considerable body of evidence has accumulated, suggesting that extended radio sources are powered quasi-continuously from the nuclei of their parent galaxies. This view is supported by the recent discovery that several radio galaxies have narrow radio jets which connect their active nuclei with the large radio lobes and which often extend for several tens of kiloparsecs. Because of their presumed association with the energy transfer processes in active galaxies, radio jets are at present being intensively studied with high-resolution radio techniques.

The closest galaxy known to have a radio jet is the giant elliptical galaxy, M87 (e.g., Wilkinson 1974), and in this case there is a well-known optical counterpart (e.g., Curtis 1918; de Vaucouleurs, Angione, and Fraser 1968). Since synchrotron decay times for electrons emitting in the optical are typically only of the order of 10 years, optical synchrotron features precisely locate the sites of energetic extranuclear interactions. Studies of optical jets such as that in M87 can, therefore, place severe constraints on the energy transfer processes involved in radio galaxies (Shklovskii 1977).

Because of this potential importance to radio source physics, we have undertaken a search for additional examples of optical synchrotron jets. We have begun the search with four galaxies known to have strong radio jets: 3C 66 B (Northover 1973), 3C 31 (Burch

\footnotetext{
${ }^{1}$ Operated by the Association of Universities for Research in Astronomy, Inc., under contract with the National Science Foundation.
}

1977), NGC 315 (Bridle et al. 1979), and 3C 449 (Perley, Willis, and Scott 1979). These objects are, on the average, 4 times farther away than M87, and have jets which are intrinsically between 2 and 10 times less powerful at $5 \mathrm{GHz}$. Thus, if they have ratios of optical-to-radio flux density similar to M87, one might expect to optically observe these jets at a level some 5 mag fainter than the M87 jet, i.e., at a total integrated blue magnitude of $B \approx 21 \mathrm{mag}$ (see de Vaucouleurs, Angione, and Fraser 1968). While difficult, the detection of such faint features on the steep gradients of stellar light in elliptical galaxies can be accomplished with modern, highly sensitive array detectors.

Inasmuch as the intergalactic medium is thought to play a significant role in determining the morphologies of extended radio sources, we expect the interstellar medium in radio jet galaxies to influence the observed properties of the jets. We have, therefore, also examined each of our four galaxies for signs of interstellar matter in the forms of optically absorbing dust clouds and hydrogen emission-line regions.

\section{OBSERVATIONS}

To make these demanding observations, we have employed the Kitt Peak ISIT vidicon video camera on the $4 \mathrm{~m}$ Mayall telescope (Robinson et al. 1979). Observations of each galaxy were obtained through a broad-band blue filter (effective wavelength $\sim 4500 \AA$, FWHM $\approx 900 \AA$ ) and through on-line and off-line $\mathrm{H} \alpha$ filters $(\mathrm{FWHM} \approx 60 \AA)$. In each case the galaxy was centered in the $70^{\prime \prime} \times 70^{\prime \prime}$ field of view of the vidicon. Integration times in the blue were $27,13,27$, 
and 41 minutes, respectively, for $3 \mathrm{C} 66 \mathrm{~B}, 3 \mathrm{C} 31$, NGC 315, and 3C 449. The $\mathrm{H} \alpha$ integration times were uniformly 13 minutes each, on-line and off-line. Stellar image profile widths in these data are 1".2 FWHM. An additional 13 minutes integration of $3 \mathrm{C} 66 \mathrm{~B}$ in the blue has not been included in the final analysis, because it was obtained through thin clouds and with poorer seeing. A second integration of $3 \mathrm{C} 31$ in the blue with poorer seeing has been used in the generation. of the background template for that galaxy (see $\S$ III) but not in the photometric analysis.

To provide an absolute flux calibration for our observations, we have observed the star HZ 4 (Oke 1974) through each of our filters. System saturation with the broad-band blue filter was avoided by recording the star's image with the telescope out of focus.

Our strategy in searching for possible optical jets in the data has been to subtract a smooth representation of the background stellar light of each galaxy, and then to examine the flattened data for features which stand out above (or below) the background. Reduction was carried out independently with the Interactive Picture Processing System (IPPS) at Kitt Peak National Observatory and the Westerbork reduction system at Leiden. Similar results were obtained. Since each galaxy has presented somewhat different problems, in $\S$ III we give an individually detailed discussion of each object.

\section{RESULTS}

\section{a) $3 C 66 B$}

This source has been studied at high resolution and at several frequencies by Northover (1973). It has a high-intensity radio jet emanating from the nucleus toward the northeast, with a total extent of $\sim 22^{\prime}$. This jet displays a considerable curvature, its position angle changing by some $40^{\circ}$ before it merges into one of the outer, relaxed components of the source. Near the nucleus, the jet consists of a number of unresolved (at about the $2^{\prime \prime}$ level) knots superposed on a smoother background component. The average strength of the jet at $5 \mathrm{GHz}$ decreases steadily away from the nucleus.

In the optical the galaxy is an elliptical classified as DE2 by Matthews, Morgan, and Schmidt (1964) and E0 by de Vaucouleurs, de Vaucouleurs, and Corwin (1976). It has a redshift of $z=0.0215$, lies near the edge of Abell 347, a richness class 0 cluster, and has two apparent companion galaxies within its outer envelope. The stellar light distribution appears somewhat distorted with a less steep gradient toward the northeast, in the direction of the jet.

An optical jet is readily evident in each of our broadband (blue) exposures (Fig. 1 [Pls. 14 and 15]). It extends out to a radius of $16^{\prime \prime}$, beyond which it disappears into the noise. Within $\sim 8^{\prime \prime}(\sim 3 \mathrm{kpc}),{ }^{2}$ the optical jet (as well as the radio jet) is significantly brighter than further from the galaxy. As is demonstrated in Figure 1,

${ }^{2}$ A value for $H_{0}$ of $75 \mathrm{~km} \mathrm{~s}^{-1} \mathrm{Mpc}^{-1}$ will be used throughout this paper. the general correspondence between the $5 \mathrm{GHz}$ and optical features is excellent, although the resolution and noise in the optical data preclude the certain identification of individual knots.

Because the stellar light distribution is slightly asymmetric, the generation of a smooth template for our flattening procedure has proved difficult. To produce Figures $1 a$ and $1 b$, we have used symmetric Hubble profiles, optimized to fit in the northeast gradient over the inner and outer parts, respectively, of the jet. In the original data one can trace the jet into a radius of $\sim 2^{\prime \prime}$, at which point the noise level has increased sufficiently to obscure the jet, if indeed it continues all the way into the nucleus.

We measure an integrated flux density for the jet in the blue of $F_{B}=9 \mu \mathrm{Jy} .^{3}$ Like M87, the optical flux density of this jet is slightly fainter than would be predicted by an extrapolation of the radio frequency spectrum into the optical.

There is a very faint, unresolved optical feature at a radius of $18^{\prime \prime}$ along the axis of the jet which could possibly be an optical knot related to the jet. No radio knot is found at this location, however, so the relation of the emission to the synchrotron jet is uncertain. There is also a $21 \mathrm{st}$ mag stellar object at $25^{\prime \prime}$ radius along the axis of the jet as defined at radii less than $\sim 10^{\prime \prime}$. It does not lie on the line of radio emission, but close to the region where the radio jet bends southward. We suppose that it is a star, unrelated to the jet. It is possible, however, that it is this star that has given rise to the claim in the literature (Minkowski, quoted by Harris and Roberts 1960; Dewhirst, quoted by Northover 1973) that the galaxy has a possible jet. On the basis of the present data, it would seem most unlikely that the jet itself would be visible, at least on the Palomar Sky Survey plates.

No evidence for dust absorption features has been found in the blue data. To search for regions of ionized hydrogen at $\sim 10^{4} \mathrm{~K}$, we have prepared our $\mathrm{H} \alpha$ filter data as follows. After subtraction of the estimated background sky levels, the on-line and offline pictures were scaled to match the signals in the outer parts of $3 \mathrm{C} 66 \mathrm{~B}$ and in the nearby companion galaxies. Then the two pictures were carefully aligned spatially to an accuracy of \pm 0.05 picture elements (i.e., $\pm 0.014^{\prime \prime}$ ) and the off-line picture subtracted from the on-line.

In the result, besides a bright, unresolved nuclear source, we find very faint emission extending $4^{\prime \prime}$ or $5^{\prime \prime}$ (some $2 \mathrm{kpc}$ at this redshift) to the southeast. This extended emission is in a direction nearly perpendicular to the axis of the jet, and its presence may be an indirect result of plasma motions which, in turn, may be responsible for the curvature of the jet (see Ford and Butcher 1979 for a discussion of the extended emission in M87). The existence of this emission is an indication not only that there is an interstellar medium in $3 \mathrm{C} 66 \mathrm{~B}$, but also that it is probably

${ }^{3}$ Conversion to $B$ magnitudes may be made via the formula $B=8.98-2.5 \log F_{B}$ (jansky), where $1 \mathrm{Jy}=$ $10^{-26} \mathrm{~W} \mathrm{~m}^{-2} \mathrm{~Hz}^{-1}$. 
inhomogeneous in nature. Further detailed spectroscopic mapping of the extranuclear emission in $3 \mathrm{C} 66 \mathrm{~B}$ is warranted.

\section{b) $3 C 31$ (NGC 383)}

High-resolution observations at $5 \mathrm{GHz}$ of this source have been made and discussed by Burch (1977). A strong radio jet is seen to emerge from the nucleus in position angle $341^{\circ}$ and to decrease in brightness with distance from the nucleus. There is evidence for an unresolved (at about the $3^{\prime \prime}$ level) knot of emission in the jet at a radius of about $4^{\prime \prime}$, beyond which the jet appears to broaden systematically before making an abrupt bend and merging with a complex outer structure. A somewhat fainter and apparently resolved counterjet is seen on the opposing side of the nucleus.

Optically, the galaxy is an elliptical or S0 (Arp 1968) at $z=0.0167$, and is part of a chain of bright galaxies. This chain, in turn, is part of a large cloud of associated galaxies extending about $9^{\circ}$ from $3 \mathrm{C} 31$ to the cluster Abell 262 (Moss and Dickens 1977).

The jet is not immediately apparent in our optical image. Instead, a ring of absorbing material is seen to encircle the nucleus at a radius of about $3^{\prime \prime}$. The ring is elliptical in shape and, from its eccentricity and variation of strength with azimuth, appears to be tilted by $\sim 35^{\circ}$ from a face-on configuration. Its presence makes removal of the background stellar light with any simple functional fit, as was used with 3C $66 \mathrm{~B}$, impossible.

Fortunately, however, the axis of symmetry of the dust lane coincides with the minor axis of the galaxy's stellar light component, and to search for an optical jet we have removed the symmetric component of the galaxy light as follows. The two available 13 minute integrations, one in somewhat inferior seeing, were first averaged. A second image was then formed by taking the mirror image about the galaxy's symmetry axis. These two pictures were then compared, point by point, and a third image was generated as the minimum value at each point. This procedure has the net effect of cleaning off any asymmetrical features, such as faint stars or a jet, which protrude above the stellar background. Finally, this cleaned template was subtracted from the original, good-seeing data frame to yield a flattened image of the galaxy.

The result is shown in Figure 2 (Pls. 16 and 17). The jet is plainly visible, extending out to $\sim 5^{\prime \prime}(\sim 1.5 \mathrm{kpc})$ from the nucleus in precisely the position angle of the strong radio jet. There is some indication that the jet does not continue all the way into the nucleus, but the increased noise level within $1^{\prime \prime}$ of the center makes this possibility uncertain. We derive an integrated (blue) flux density for the jet of $F_{B}=6.2 \mu \mathrm{Jy}$.

Again, this flux is less than one would predict from an extrapolation of the radio spectrum, and also slightly less than the scaled flux from the M87 jet.

We note in passing that the angle between the jet's axis and the minor axis of the dust ring is $65^{\circ} .5$, which makes it improbable that the rotation axis of the ring has any relevance to the radio source. The situation, therefore, is unlike that in the sources discussed by Kotanyi and Ekers (1979), where the absorption bands and the radio axes are nearly perpendicular. Also, the jet appears to cross the dust ring, but from the geometry, one cannot tell whether the jet is approaching or receding.

Our $\mathrm{H} \alpha$ observations of this galaxy reveal an unresolved nuclear source, but provide no evidence for any extended emission. The presence of the dust ring, however, clearly demonstrates the existence of an interstellar medium in the galaxy. That the optical emission occurs at very nearly the same radial distance as the dust may be significant.

\section{c) $N G C 315$}

This giant radio galaxy has been studied at high resolution by Bridle et al. (1979). It possesses a strong jet to the northwest of the nucleus and a somewhat weaker counterjet to the southeast. At $5 \mathrm{GHz}$, the main jet begins at about 7 " from the nucleus, maintains an approximately constant brightness for about $10^{\prime \prime}$, and then quickly decreases in intensity as it broadens. For the first $10^{\prime \prime}$ of its length, it appears unresolved (at the $2^{\prime \prime}$ level) perpendicular to its axis. Near the nucleus, the morphology of this jet resembles that of the $3 C 31$ jet.

In the optical, NGC 315 is a relatively isolated elliptical or S0 (de Vaucouleurs, de Vaucouleurs, and Corwin 1976) galaxy without any obviously distinguishing properties. We have employed both types of template generation procedures discussed previously, but have found no evidence for any optical counterpart to either radio jet. Assuming any optical features to be unresolved in their transverse dimension and not significantly longer than $10^{\prime \prime}$ in length, we derive an integrated flux density limit of $F_{B}<3.2 \mu \mathrm{Jy}$.

We do find a series of five or six discrete absorption clouds aligned in an arc just to the west of the nucleus, at distances ranging from 3-7". The largest two of these clouds appear to be marginally resolved, and if we assume a gas-to-dust ratio similar to the Galaxy's (Jenkins and Savage 1974), we can derive a lower limit to their masses of $\sim 10^{6} M_{\odot}$. One of these clouds lies projected onto the axis of the jet at about $3^{\prime \prime}$ from the nucleus. Finally, we find a weak, unresolved nuclear $\mathrm{H} \alpha$ source in our narrow-band data, but do not detect any convincing signs of extended emission.

\section{d) $3 C 449$}

The galaxy 3C 449 has recently been studied at high resolution at 1.4 and $5 \mathrm{GHz}$ by Perley, Willis, and Scott (1979). Two opposing jets of nearly equal intensities are found. They begin about $10^{\prime \prime}$ from the nucleus and steadily widen before fanning out abruptly at a radius of 1 ' to form outer complexes that appear relaxed. The two jets show considerable internal structure, with numerous condensations superposed on a smoother ridgeline of emission. Whether these condensations include high surface brightness, unresolved knots of the kind found in the other objects, is not entirely clear, but the indication 
is that all features are at least marginally resolved at the 1-2" level.

Optically, the galaxy has been classified a cD elliptical by Wyndham (1966) and an S0 by de Vaucouleurs, de Vaucouleurs, and Corwin (1976). It lies in an interacting group of galaxies at $z=0.0171$. We find no evidence for either of the two jets in our data. We assign an upper limit to the brightness of each of these jets of $F_{B}<2.5 \mu \mathrm{Jy}$, subject to the same length and width restrictions as for the NGC 315 jet.

Much like that seen in 3C 31, we find an apparently tilted ring of absorbing matter encircling the nucleus of $3 C 449$. The ring has a major axis diameter of about $2^{\prime \prime}$ roughly in P.A. $135^{\circ}$, and is strongest to the southwest of the nucleus. In the light of $\mathrm{H} \alpha$ an unresolved nuclear source is seen. There is also a hint in our data of a short $\mathrm{H} \alpha$ extension to the south of the nucleus, but the presence of the dust ring together with slightly different seeing in the on-line and off-line data makes this conclusion very uncertain.

\section{DISCUSSION}

\section{a) Intercomparison of Galaxies That Have Jets}

Too few radio galaxies with jets have been surveyed optically to allow a detailed statistical study of their properties. However, we feel it is instructive here to compare the properties of the few cases for which data are available in the hope that strong trends might become apparent and provide some clues to the mechanisms at work. In Table 1 we list some relevant parameters of the galaxies which we have observed and, for comparison, of M87 and 3C 273, the only other radio sources with well-studied jets. In addition to the observed parameters of the jets, we have also included some parameters which might be related to the past and present activity of the galaxies, such as the total optical and radio luminosities, the radio luminosity of the core, and the linear size of the radio galaxy. Most of the columns in Table 1 are selfexplanatory. The flux densities and luminosities of the jets per kiloparsec are given as possible comparative estimates for the energy dissipated or transported along the jets. Their derivation is at present somewhat subjective.

The radio spectral indices $\alpha$ in Table 1 are defined by $F_{v} \propto \nu^{\alpha}$ and have uncertainties that are typically $\sim 0.1$. Note that these indices may be affected by spectral curvature: for the knots in M87 there is evidence that the spectra flatten significantly below $1600 \mathrm{MHz}$ (Wilkinson 1974). The radio-optical spectral index $\left(\alpha_{\mathrm{rad}}{ }^{\mathrm{opt}}\right)$ has been derived in the radial interval $1-2 \mathrm{kpc}$ along the jets. This particular interval is chosen rather arbitrarily for the purpose of comparison with the M87 jet, which is brightest at these radii. No value for $\alpha_{\mathrm{rad}}{ }^{\text {opt }}$ is given for 3C 449, because both gaps in this source extend beyond $2 \mathrm{kpc}$. At a radius of some $5 \mathrm{kpc}$, however, there is emission, and we can set a limit of $\alpha_{\mathrm{rad}}{ }^{\text {opt }}<-0.7$. Because of the large range in frequency involved, the radio-optical spectral indices are relatively insensitive to errors in the radio or optical flux densities. We estimate their uncertainties to be $\sim 0.03$.

Perley, Willis, and Scott (1979) have drawn attention to the "gaps" which exist between the radio cores and the start of the radio jets in several sources. Since these gaps may be connected with the efficiency of the energy

TABLE 1

Observed Properties of Radio Jets

\begin{tabular}{|c|c|c|c|c|c|c|}
\hline GALAXY & M87 & $3 \mathrm{C} 66 \mathrm{~B}$ & $3 \mathrm{C} 31$ & NGC. 315 & $3 C 449$ & $3 \mathrm{C} 273$ \\
\hline 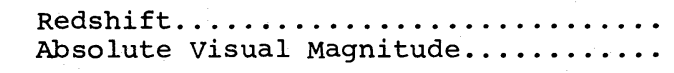 & $\begin{array}{l}0.0043 \\
-22.8\end{array}$ & $\begin{array}{l}0.0215 \\
-21.8\end{array}$ & $\begin{array}{l}0.0163 \\
-22.0\end{array}$ & $\begin{array}{l}0.0167 \\
-21.6\end{array}$ & $\begin{array}{l}0.0171 \\
-21.0\end{array}$ & $\begin{array}{l}0.158 \\
-26.1\end{array}$ \\
\hline $\begin{array}{l}\text { RADIO SOURCE } \\
\text { LOg Luminosity }\left(\mathrm{W} \mathrm{m}^{-\mathbf{a}} \mathrm{Hz}^{-1}\right)\end{array}$ & & & & & & \\
\hline 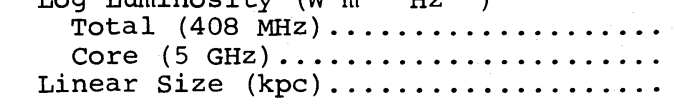 & $\begin{array}{l}25.26 \\
23.15 \\
80\end{array}$ & $\begin{array}{l}25.20 \\
23.23 \\
150\end{array}$ & $\begin{array}{l}24.70 \\
22.81 \\
570\end{array}$ & $\begin{array}{l}(24.26) * \\
23.54 \\
1000\end{array}$ & $\begin{array}{l}24.60 \\
22.43 \\
290\end{array}$ & $\begin{array}{l}27.36 \\
27.24 \\
42\end{array}$ \\
\hline \multicolumn{7}{|l|}{ JET } \\
\hline $\begin{array}{l}\text { Length Radio Jet }(\mathrm{kpc}) \ldots \ldots \ldots \ldots \ldots \ldots \\
\text { Central Radio Gap }(\mathrm{kpc}) \ldots \ldots \ldots \ldots \ldots \ldots \\
\text { Log Flux Density } \mathrm{kpc}^{-1}+\left(\mathrm{w}_{\mathrm{m}}^{-} \mathrm{Hz}^{-i}\right)\end{array}$ & $\begin{array}{l}1.5 \\
0.3\end{array}$ & $\begin{array}{r}50 \\
<1.5\end{array}$ & $\begin{aligned} & 17 \\
< & 0.7\end{aligned}$ & $\begin{array}{c}300 \\
2\end{array}$ & $\begin{array}{c}20 \\
3\end{array}$ & $\begin{array}{l}(42) \\
---\end{array}$ \\
\hline 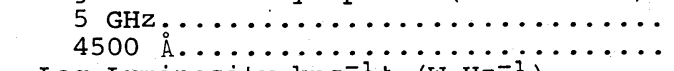 & $\begin{array}{l}-25.28 \\
-28.68\end{array}$ & $\begin{array}{l}-27.13 \\
-31.16\end{array}$ & $\begin{array}{l}-27.70 \\
-31.33\end{array}$ & $\begin{array}{r}-28.22 \\
<-32\end{array}$ & $\begin{array}{r}<-28.6 \\
<-32\end{array}$ & -31.91 \\
\hline Log Luminosity $\mathrm{kpc}^{-1}+\left(\mathrm{W} \mathrm{Hz}^{-1}\right)$ & & & & & & \\
\hline 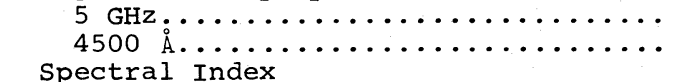 & $\begin{array}{l}23.27 \\
19.87\end{array}$ & $\begin{array}{l}22.81 \\
18.77\end{array}$ & $\begin{array}{l}22.00 \\
18.37\end{array}$ & $\begin{aligned} & 21.50 \\
< & 17.7\end{aligned}$ & $\begin{array}{l}<21.1 \\
<17.75\end{array}$ & 19.70 \\
\hline 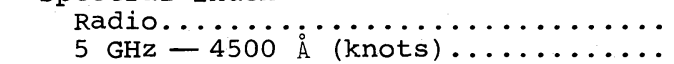 & $\begin{array}{l}-0.55 \\
-0.65\end{array}$ & $\begin{array}{l}(-0.5) \neq \\
-0.70\end{array}$ & $\begin{array}{l}-0.4 \\
-0.72\end{array}$ & $\begin{aligned} &-0.6 \\
&<-0.74\end{aligned}$ & -0.6 & --- \\
\hline
\end{tabular}

Intrinsic parameters are calculated using $\mathrm{H}_{0}=75 \mathrm{~km} \mathrm{~s} \mathrm{Mpc}^{-1}, \mathrm{q}_{0}=1$.

*Extrapolated from Bridle et al. 1979.

tThe flux density $\mathrm{kpc}^{-1}$ is, where possible, estimated in the region $1-2 \mathrm{kpc}$ from the nucleus. fIncludes contribution from core. 
transport or the frequency of nuclear activity, we give estimates for their projected dimensions in Table 1 .

For completeness, 3C 273 has been included in Table 1 (using data from Kron, Ables, and Hewitt 1972), but we note that the energy outputs for both the optical QSO and the radio source are several orders of magnitude greater than those for the galaxies. Also, there are insufficient radio data available along the length of the jet to make detailed comparison with the optical emission. It is interesting to note, however, that the luminosity per kiloparsec of the optical jet in 3C 273 is comparable with that in M87.

There are then several possible trends apparent in Table 1 which should be considered as more data become available. Relatively flatter radio-optical spectra and larger jet luminosities seem to be associated with larger total optical and radio luminosities and smaller radio gaps.

Although the differences in $\alpha_{\mathrm{rad}}{ }^{\text {opt }}$ are significant, it is remarkable that of the five galaxies with radio jets that have been surveyed, the $\alpha_{\mathrm{rad}}{ }^{\text {opt }}$ for three of them lie within 0.07 , and imply spectra only slightly steeper than those at radio frequencies. The limit on $\alpha_{\text {rad }}$ opt for NGC 315 is consistent with similar behavior. This implies that the spectra of jets probably extend continuously from $\sim 10^{9}$ to $\sim 10^{15} \mathrm{~Hz}$, and are almost straight. (Note, however, that the spectrum of the knots in the M87 jet may steepen either in the optical [Ables and Kron 1973] or in the infrared [Kinman, Grasdalen, and Rieke 1974].) It has recently been suggested that a nonthermal spectrum extending over such a wide frequency range can be produced by the Fermi mechanism (Blandford and Königl 1979; Blandford and Ostriker 1978; Bell 1978).

Both of the optically undetected jets here have spectral indices between 1.4 and $5 \mathrm{GHz}$ slightly larger than the two undetected jets. This difference could be a useful clue in determining which radio jets can be seen in the optical and may be a consequence of the different regimes of the particle acceleration processes in progress.

\section{b) Relationship to Physics of Jets}

We now consider various properties of the jets that may affect their luminosities and ratios of optical-toradio powers.

\section{i) Angle of the Jet to the Line of Sight}

If the jets represent outflow at relativistic velocities, one would anticipate a dependence of intensity on aspect (see Rees 1978; Blandford and Königl 1979). We see only two possible methods of determining the aspect angle of a given jet. The first relies on the chance occurrence of a favorable geometry involving a uniform dust ring, such as the one in $3 \mathrm{C} 31$. If a jet were seen to cross such a ring on the near side, one would have clear evidence that it is approaching. Unfortunately, although the $3 \mathrm{C} 31$ jet does appear to cross in front of the ring, it crosses the rear part of the ring and hence provides no unambiguous evidence as to its orientation. The second method assumes that all jets are in reality opposing double features of equal intrinsic strength, whose observed relative brightnesses are determined by the relativistic Doppler effect. In this case, a relationship between the presence and strength of the optical features and the relative strengths of the radio jets might be expected. Unfortunately, the present data set does not support such a simple hypothesis; the two sources without optical jets, NGC 315 and 3C 449, have the largest and smallest ratios respectively between opposing radio jets. This situation, together with some indications that the flow velocities in radio jets may be only of the order $10^{3} \mathrm{~km} \mathrm{~s}^{-1}$ (Blandford and Icke 1978; Perley, Willis, and Scott 1979), leads us at this point to view this explanation of the differences between jets as unattractive.

\section{ii) Amount of Energy Being Conducted to the Lobes}

A dependence of the observed intensity of a jet on the flux of energy being transported outward would be consistent with the tendency of the jets with relatively high optical power to be associated with larger total radio luminosities. These more luminous sources have presumably had a larger integrated activity in the past and must, therefore, be supplied with more energy. From the radio luminosity of the cores, there is no evidence that the galaxies are more active at present, but the highly energetic processes required to generate optical radiation in the jets may vary sporadically, as a result of unsteady nuclear activity (Rees 1978).

\section{iii) Efficiency of the Energy Transport}

It is possible that higher jet luminosities and smaller radio gaps indicate a lower efficiency of energy transfer. Then, for example, a larger fraction of the transported energy in the M87 jet would be dissipated outward along its path, compared with those fractions in NGC 315 or $3 C 449$. In a source where the energy transport is less efficient, most of the energy would be dissipated close to the galaxy, and the corresponding radio source might well be smaller on the average.

It is not clear what processes will govern the efficiency of energy transport. Inefficiency may be related to the degree to which the energy transfer is relativistic (Rees 1978) or to the amount of interstellar matter entrained in the beam (Blandford and Königl 1979). Some evidence that the latter mechanism may be important is provided by the tendency of the jet luminosity and $\alpha_{\mathrm{rad}}$ opt to depend on the absolute optical magnitude of the galaxy. If optically luminous galaxies have more and denser interstellar matter, the jets might well be caused to radiate more intensely.

Each of the four galaxies we observed has a significant amount of interstellar matter, and this matter seems to be very clumped. We would suppose that each of the three galaxies with visible dust absorption regions also possesses a hot, invisible interstellar medium, much like the two-phase medium in our Galaxy. If the extended $\mathrm{H} \alpha$ emission seen in the fourth galaxy, 3C $66 \mathrm{~B}$, is found to be similar to the extended emission in M87, then that object too probably has a 
nonuniform interstellar medium. Because it is highly likely that jets will interact with any interstellar medium, recent models of jets involving shock waves driven into inhomogeneities in the medium (Blandford and Königl 1979) seem particularly appropriate.

\section{c) Conclusions}

Our observations suggest that the presence of optical jets could be a fairly general property of galaxies with radio jets. It is important both to search for optical jets in a larger sample and to make more sensitive observations of cases that have already been detected. Determination of the optical colors and polarizations, the ratio of optical-to-radio emission, and the nature of the interstellar medium as a function of distances from galactic nuclei could provide severe constraints on the energy transport mechanism. Such measurements are feasible with instruments which are already in use or will shortly become available. In particular, observations in the ultraviolet would be several times more sensitive to nonthermal optical emission in galaxies than the blue sensitive measurements reported here.

We thank Professor Oort for his useful comments on the manuscript. G. K. M. wishes to acknowledge support from NATO grant 1828.

Ables, H. D., and Kron, G. E. 1973, Ap. J., 181, 19.

\section{REFERENCES}

Arp, H. C. 1968, Pub. A.S.P., 80, 129.

Bell, A. R. 1978, M.N.R.A.S., 182, 147 and 443.

Blandford, R. D., and Icke, V. 1978, M.N.R.A.S., 185, 527.

Blandford, R. D., and Königl, A. 1979, Ap. Letters, 20, 15.

Blandford, R. D., and Ostriker, J. P. 1978, Ap. J. (Letters), 221, L29.

Bridle, A. H., Davis, M. M., Fomalont, E. B., Willis, A. G., and Strom, R. G. 1979, Ap. J. (Letters), 228, L9.

Burch, S. F. 1977, M.N.R.A.S., 181, 599.

Curtis, H. D. 1918, Pub. Lick Obs., 13, 31.

de Vaucouleurs, G., Angione, R., and Fraser, C. W. 1968, Ap. Letters, 2, 141.

de Vaucouleurs, G., de Vaucouleurs, A., and Corwin, $\mathbf{H}$. 1976, Second Reference Catalogue of Bright Galaxies (Austin: University of Texas Press).

Ford, H. C., and Butcher, H. 1979, Ap. J. Suppl., in press

Harris, D. E., and Roberts, J. A. 1960, Pub. A.S.P., 72, 237

Jenkins, E. B., and Savage, B. D. 1974, Ap. J., 187, 243.

Kinman, T. D., Grasdalen, G. L., and Rieke, G. H. 1974, Ap. J. (Letters), 194, L4.

Kotanyi, C. G., and Ekers, R. D. 1979, Astr. Ap., 73, L1.

Kron, G. E., Ables, H. D., and Hewitt, A. V. 1972, Pub. A.S.P., 84, 303.

Matthews, T. A., Morgan, W. W., and Schmidt, M. 1964, Ap. J., 140, 35

Moss, C., and Dickens, R. J. 1977, M.N.R.A.S., 178, 701

Northover, K. J. E. 1973, M.N.R.A.S., 165, 369.

Oke, J. B. 1974, Ap. J. Suppl., 27, 21.

Perley, R. A., Willis, A. G., and Scott, J. S. 1979, Nature, 281, 437.

Rees, M. J. 1978, M.N.R.A.S., 184, 61P.

Robinson, W., Ball, W., Vokac, P., Piegorsch, W., and Reed, R. 1979, in Proc. SPIE Symposium, Instrumentation in Astronomy, No. 3, Vol. 172, p. 98.

Shklovskii, I. S. 1977, Soviet Astr.-AJ, 21, 401

Wilkinson, P. N. 1974, Nature, 252, 661.

Wyndham, J. D. 1966, Ap. J., 144, 459.

HaRVey R. Butcher: Kitt Peak National Observatory, P.O. Box 26732, Tucson, AZ 85726

GeOrge K. Miley and Wil van Breugel: Sterrewacht Leiden, 2300 RA Leiden, The Netherlands 
PLATE 14
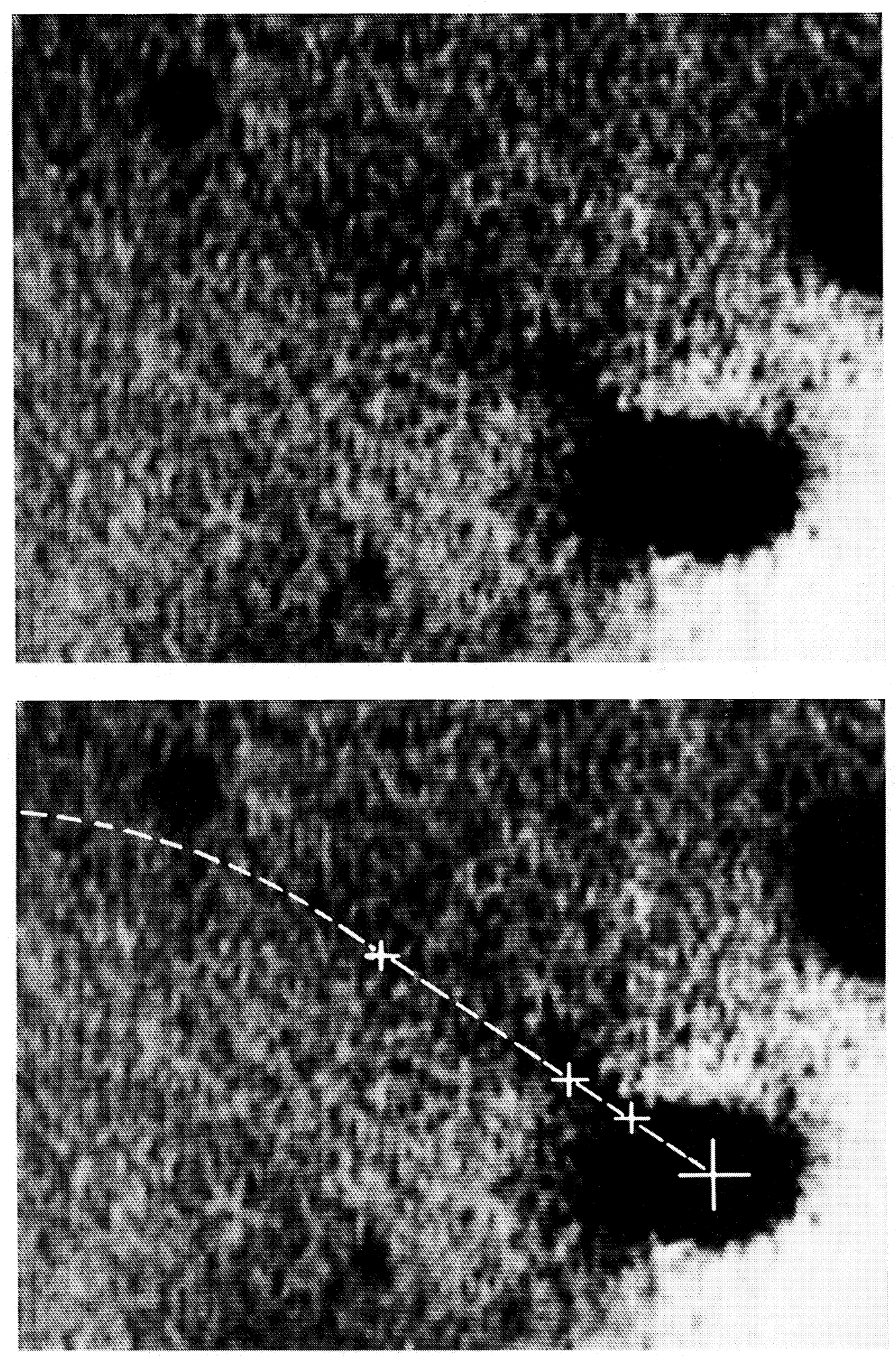

Fig. 1.-Two representations of the data for the $3 \mathrm{C} 66 \mathrm{~B}$ optical jet.

Fig. 1a.-The upper panel shows the outer part of the jet. The lower panel shows the same data with the positions of the nuclear radio source, the principal radio knots, and the ridgeline of radio emission in the jet overlaid.

BUTCHER et al. (see page 750) 


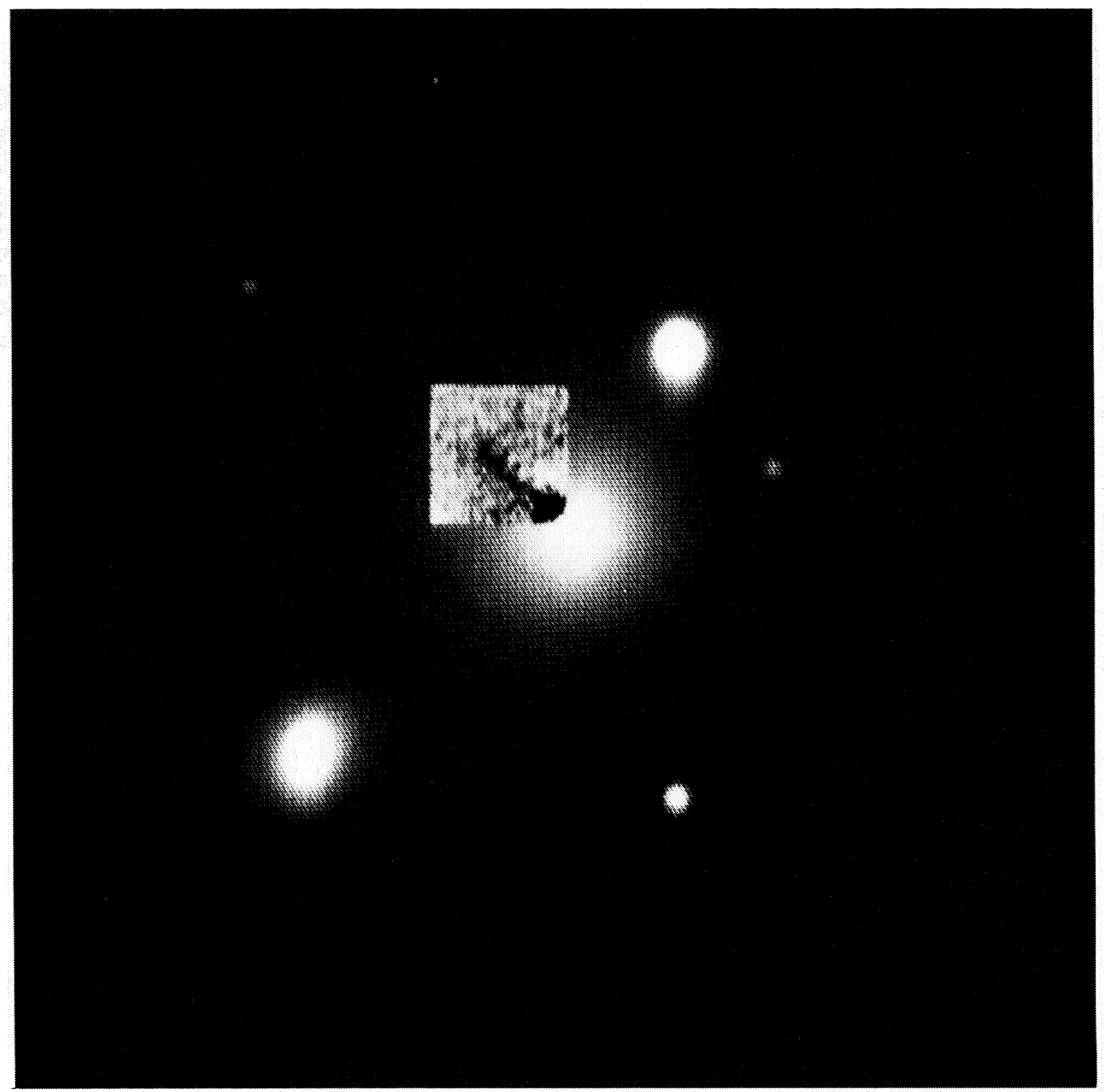

FIG. 1b. - The bright, inner part of the jet is shown here, reinserted into the average of original vidicon data frames. The inserted region has had its background starlight subtracted and its relative contrast greatly enhanced. North is at the top and east is to the left in these data. The scale is given approximately by the $25^{\prime \prime}$ distance from the nucleus to the 21 st mag star to the northeast along the axis of the jet.

BUTCHER et al. (see page 750) 
PLATE 16

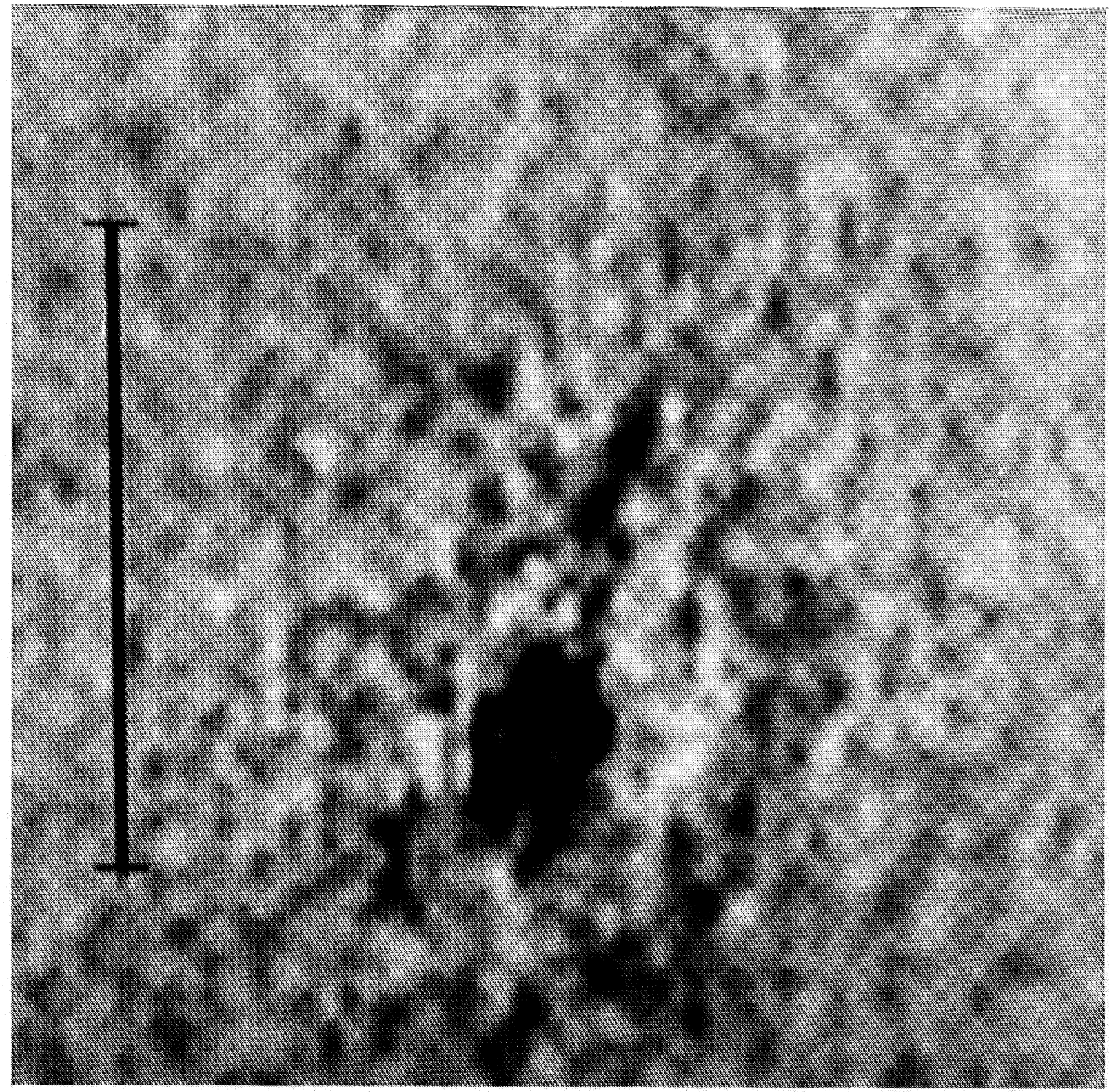

FIG. 2.-Two displays of the optical jet in 3C 31 .

FIG. $2 a$. - The raw data after subtraction of the background template (see text). The optical jet is seen crossing the imperfectly subtracted dust ring at P.A. $341^{\circ}$. North is at the top and east to the left. The error bar is $10^{\prime \prime}$ in length. Note that the nucleus of the galaxy is in reality $1^{\prime \prime}$ or so north of the midline of the central black feature seen here.

Butcher et al. (see page 751) 


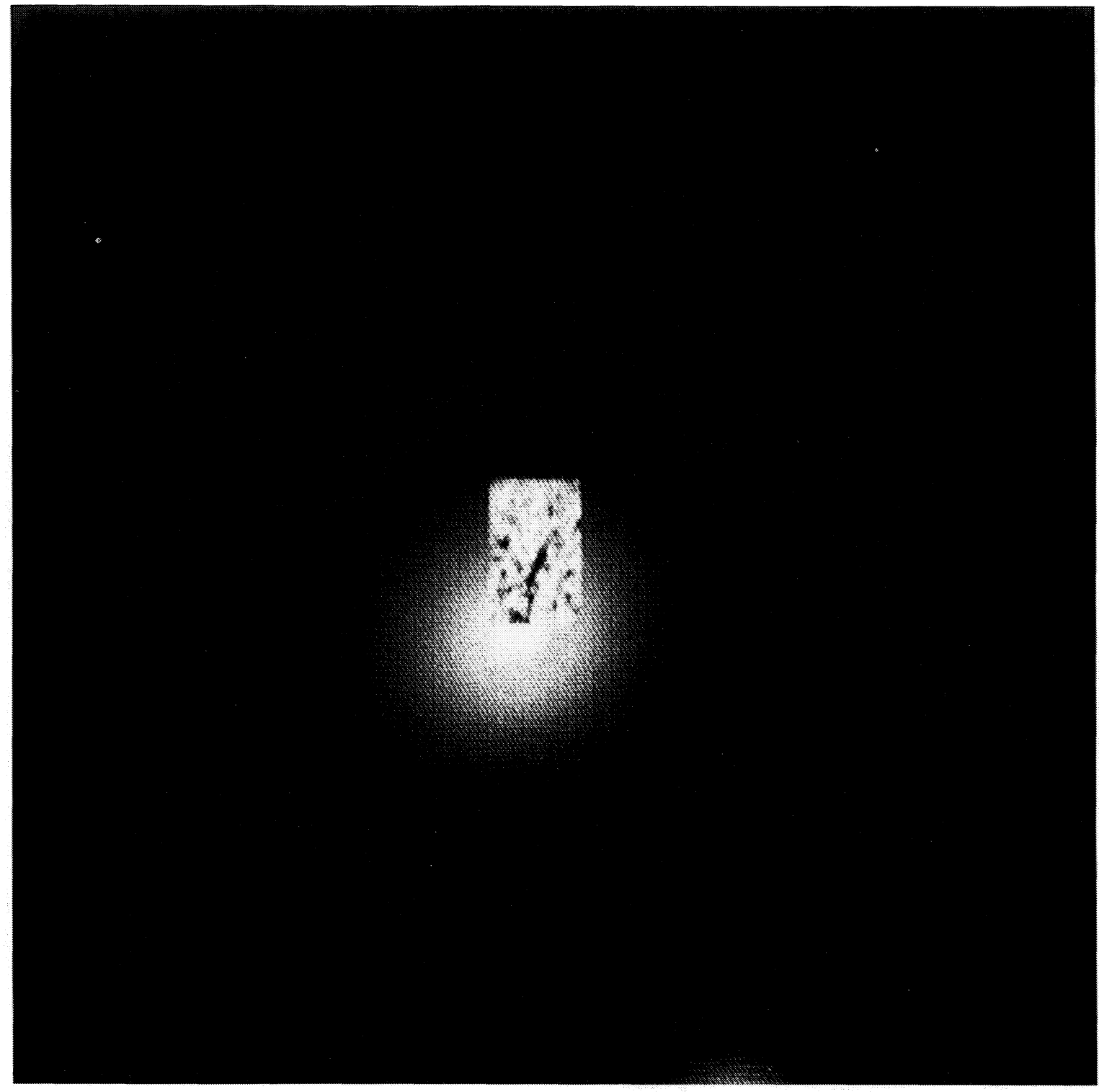

FIG. $2 b$. - The background-subtracted image of the optical jet reinserted into the original vidicon data frame. Its contrast is greatly enhanced relative to that of the galaxy.

BUTCHER et al. (see page 751) 